Royal Netherlands Institute for Sea Research

This is a postprint of:

Bale, N.; de Vries, S.; Hopmans, E.C.; Sinninghe Damsté, J.S. \& Schouten, S. (2017). A method for quantifying heterocyst glycolipids in biomass and sediments. Organic Geochemistry, 110, 33-35

Published version: $\underline{\mathrm{dx} . \text { doi.org/10.1016/j.orggeochem.2017.04.010 }}$

Link NIOZ Repository: www.vliz.be/imis?module=ref\&refid=285872

[Article begins on next page]

The NIOZ Repository gives free access to the digital collection of the work of the Royal Netherlands Institute for Sea Research. This archive is managed according to the principles of the Open Access Movement, and the Open Archive Initiative. Each publication should be cited to its original source - please use the reference as presented.

When using parts of, or whole publications in your own work, permission from the author(s) or copyright holder(s) is always needed. 


\section{A method for quantifying heterocyst glycolipids in biomass}

\section{2 and sediments}

3 Nicole Bale ${ }^{a^{*}}$, Steven de Vries ${ }^{a}$, Ellen C. Hopmans ${ }^{a}$, Jaap S. Sinninghe Damstéa,b,

4 Stefan Schouten ${ }^{a, b}$

$5 \quad$ a NIOZ Royal Institute for Sea Research, Department of Marine Microbiology and

6 Biogeochemistry, and Utrecht University, P.O. Box 59, 1790 AB Den Burg, Texel,

$7 \quad$ The Netherlands

$8 \quad$ b Department of Earth Sciences, Faculty of Geosciences, Utrecht University, PO

9 Box 80.121, 3508 TA Utrecht, The Netherlands

10

* Corresponding author: nicole.bale@nioz.nl (Nicole Bale).

11 ABSTRACT

Heterocyst glycolipids (HGs) are biomarkers for heterocystous $\mathrm{N}_{2}$ fixing cyanobacteria and consist of a sugar moiety bound to an even-numbered $\mathrm{C}_{26}-\mathrm{C}_{32}$ alkyl chain, containing various alcohol or ketone groups. They have been reported in ancient sediments but, due to a lack of standards, they were quantified in terms of integrated peak area response. Here we describe the use of a commercially available glycolipid (n-dodecyl-B-D-glucopyranoside) as an internal standard (IS) for reliable quantitative analysis. The common HG 1-(O-

19 hexose)-3,25-hexacosanediol was isolated using both normal and reversed phase chromatography and used to assess the relative response factor compared with the IS, allowing correction of difference in mass spectral response between the IS and the target HGs.

24 Keywords: Heterocyst, Cyanobacteria, heterocyst glycolipids, semi-preparative 
high performance liquid chromatography, quantification

\section{Introduction}

In all heterocyst-forming cyanobacteria, the heterocyst cell envelope contains heterocyst glycolipids (HGs), comprising either a hexose or pentose head group, bound to long chain diols, triols, or hydroxyketones (Gambacorta et al., 1995, 1999; Bauersachs et al., 2009, 2011; Woermer et al., 2012; Schouten et al., 2013; Bale et al., 2015). Recent studies have applied specific high performance liquid chromatography-multistage mass spectrometry (HPLC-MS²) methods to detect and characterize HGs in cyanobacterial cultures and samples from the natural environment (Bauersachs et al., 2009, 2014; Woermer et al., 2012; Schouten et al., 2013; Bale et al., 2015, 2016). However, the methods were not used for absolute quantification of the HGs due to the lack of authentic standards. Here we describe the application of a commercially available short chain glycolipid as an internal standard (IS), which allows absolute quantification of $\mathrm{HGs}$ in biomass and sediments. To correct for differences in response factor between the IS and naturally occurring HGs, we isolated a HG from an extract of mixed biomass material of heterocystous cyanobacteria. This was used with the IS to constrain the relative response factor (RRF).

43 Furthermore, instead of HPLC-MS² in multiple reaction mode (MRM) we used a UHPLC (ultra high pressure liquid chromatography) separation combined with high resolution mass spectrometry (HRMS) in full scan mode to quantify HGs using this novel approach.

\section{$47 \quad 2 . \quad$ Experimental}

The IS was n-dodecyl-B-D-glucopyranoside ( $\geq 98 \%$; Sigma-Aldrich, 
Zwijndrecht, The Netherlands). For isolation of a hexose-containing $\left(\mathrm{C}_{6}\right) \mathrm{HG}$

50

51

52

53

54

55

56

57

58

59

60

61

62

63

64

65

66

67

68

69

70

71

72

standard, freeze dried cyanobacterial biomass (0.14 g dry wt; comprising a mixture of cultivated Anabaena sp. (CCY 9922) and Nostoc sp. (CCY 1933); culture conditions detailed by Bauersach et al. 2009) and was extracted using a modified Bligh-Dyer extraction as described by Bale et al. (2015). Initial analysis of the extract and all further analyses, unless specified otherwise, were carried out using UHPLC-HRMS (Q-Exactive Orbitrap; Thermo Scientific, Waltham, MA), as described by Moore et al. (2016) with some modifications: (i) An Acquity UPLC BEH HILIC column (1.7 $\mu \mathrm{m}, 2.1$ x 150 mm; Waters) was used; (ii) the elution program was $100 \% \mathrm{~A}$ at $5 \mathrm{~min}, 66 \% \mathrm{~A}$ at $25-40 \mathrm{~min}, 40 \% \mathrm{~A}$ at $55 \mathrm{~min}$, $30 \% \mathrm{~A}$ at $65 \mathrm{~min}$, where $\mathrm{A}=$ hexane/propan-2-ol/ $\mathrm{HCO}_{2} \mathrm{H} / 14.8 \mathrm{M} \mathrm{NH}_{3 a q}$ (79:20:0.12:0.04, v/v) and $\mathrm{B}=$ propan-2-ol/ $\mathrm{H}_{2} \mathrm{O} / \mathrm{HCO}_{2} \mathrm{H} / 14.8 \mathrm{M} \mathrm{NH}_{3 \mathrm{aq}}$ (88:10:0.12:0.04, v/v). The total run time was $70 \mathrm{~min}$ with a re-equilibration period of 20 min between runs.

Analysis of the HGs in the cyanobacterial extract revealed that 1-(Ohexose)-3,25-hexacosanediol (hereafter $\mathrm{C}_{6} \mathrm{HG}_{26}$ diol) was the most abundant $\mathrm{HG}$ and was therefore targeted for isolation. It was isolated, first using the semipreparative HPLC system, fraction collector, column, eluent composition and elution program described by Bauersachs et al. (2009). The column effluent was collected in 1 min fractions and the fractions containing the target HGs were pooled and purified in reverse phase with a semi-preparative Symmetry $\mathrm{C}_{18}$ column (250 x 10 mm, 7 pm; Waters, Milford, MA). Typical injection volume was $200 \mu \mathrm{l}$, containing up to $1 \mathrm{mg}$ of extract. The gradient and conditions were adapted from Wörmer et al. (2013): eluent $\mathrm{A}$ was $\mathrm{MeOH} / \mathrm{H}_{2} \mathrm{O}(85: 15$, v/v) and 
eluent B MeOH/propan-2-ol (50:50, v/v), at $3 \mathrm{ml} / \mathrm{min}$. The elution program was $100 \% \mathrm{~A}$ for $2 \mathrm{~min}$, then an increase to $15 \% \mathrm{~B}$ at $2.10 \mathrm{~min}$, then to $43.5 \% \mathrm{~B}$ at 15 min (held to $16 \mathrm{~min}$ ), and subsequent washing with $100 \%$ B from 16 min to 24 min before returning to $100 \% \mathrm{~A}$ for $5 \mathrm{~min}$. The effluent was collected in $0.25 \mathrm{~min}$ fractions, between 7 and $15 \mathrm{~min}$.

Fractions from semi-preparative HPLC were screened for the presence of the target HGs with flow injection analysis using linear ion trap MS (Thermo Scientific LTQ XL, Ion Max source) with electrospray ionization (ESI); source settings were as described by Moore et al. (2016) in selected reaction monitoring $(\mathrm{SRM})$ mode, targeting the dominant parent $\rightarrow$ product transition for the $\mathrm{C}_{6} \mathrm{HG}_{26}$ diol: $m / z 577 \rightarrow m / z 415$.

Final combined fractions were analyzed using UHPLC-HRMS, with conditions as described above for initial extract analysis. This suggested that the final combined fraction was pure, i.e.no other peaks were present in the base peak chromatogram. $\mathrm{C}_{6} \mathrm{HG}_{26}$ diol was present as two isomers (annotated as $\mathrm{HG}$ and $\mathrm{HG}^{\prime}$ in Fig. 1a), with the same exact mass and same fragmentation in $\mathrm{MS}^{2}$, so it was surmised that their hexose moieties represented two diastereoisomers. The ratio of the isomer peak areas remained constant at $1.9 \pm 0.13$ in all analyses. Their peak areas were combined and they were treated as a single component. The relative response factor (RRF) of the $\mathrm{C}_{6} \mathrm{HG}_{26}$ diol vs. the synthetic glycolipid IS was determined using the same analytical method by injection of mixtures of the $\mathrm{C}_{6} \mathrm{HG}_{26}$ diol and IS (1:1, w/w) in amounts ranging from 1 to $10 \mathrm{ng}$ on column.

A microbial mat was sampled from a beach on the North Sea Dutch 
barrier island of Schiermonnikoog in April 2010 (Station III in Fan et al., 2015)).

98

99

100

101

102

103

104

105

106

107

108

109

110

111

112

113

114

115

116

117

118

119

120

Extraction of the mat was carried out as described for sediments by Bale et al. (2015) with addition of the IS $(2 \mu \mathrm{g} / \mathrm{ml})$ before filtration of the extract through a $0.45 \mu \mathrm{m}$ regenerated cellulose syringe filter (4 mm diameter; Grace Alltech,

Deerfield, IL). Analysis of the sample was carried out using UHPLC-HRMS as described above.

\section{Results and discussion}

N-dodecyl-B-D-glucopyranoside was selected as an internal standard as it is a commercially available analogue of the naturally occurring glycolipids, yet contains a carbon chain not observed in nature. The MS ${ }^{2}$ spectrum (Fig. 1b), resulting from fragmentation of $\left[\mathrm{M}+\mathrm{NH}_{4}\right]^{+}$. at $m / z 366$ also contained the $[\mathrm{M}+\mathrm{H}]^{+}$ protonated molecule at $m / z 349$ and exhibited characteristic fragment ions at $m / z 180\left(\left[\mathrm{M}+\mathrm{NH}_{4} \text {-dodecanol }\right]^{+}\right)$and $m / z 163\left([\mathrm{M}+\mathrm{H} \text {-dodecanol }]^{+} \cdot\right)$. Sequential losses from $m / z 163$ of $\mathrm{OH}$ groups as water, at $m / z 145$ and $m / z 127$, were also observed. The pattern is in contrast to that observed for HGs (Bauersachs et al., 2009), where the dominant fragment ions in $\mathrm{MS}^{2}$ correspond to loss of a hexose moiety and further losses of water therefrom. Hence, while the IS is suitable for quantification in full MS, its fragmentation pattern does make it less suitable for quantification of $\mathrm{HGs}_{\mathrm{s}}$ in $\mathrm{MS}^{2}$, e.g. for a selected reaction monitoring (SRM) method. However, the use of HRMS affords similar specificity and sensitivity in full scan mode compared with $\mathrm{MS}^{2}$-assays on mass spectrometers with nominal mass resolution. Furthermore, quantification in full scan has the benefit that factors such as differing fragment yield, as is the case with MRM, no longer play a role. 
122 naturally occurring HGs due the lack of $\mathrm{OH}$ groups on the dodecyl chain. To

123 correct for the difference, we isolated a natural HG from an extract of mixed

124 biomass material of heterocystous cyanobacteria. Following a two-step procedure

125 using semi-preparative HPLC, 0.506 $\mathrm{mg}$ of the $\mathrm{C}_{6} \mathrm{HG}_{26}$ diol was isolated. This

126 HG commonly occurs in sediments (Bauersachs 2010) and we assume that other

127 HGs have similar MS response. Response curves for the IS and the $\mathrm{C}_{6} \mathrm{HG}_{26}$ diol

128 were determined at a range of concentration (1 - $10 \mathrm{ng}$ on column; Fig. 1c) and

129 the slopes used to determine the RRF for the two HGs. The concentration range

130 was chosen firstly to reflect the peak areas in samples from the natural

131 environment when analyzed using UHPLC-HRMS (N.B., unpublished) and

132 secondly as this was range which produced a linear response. In order to

133 examine its variability RRF, was determined 3x over a period of a month and fell

134 in the range $2.1-3.0$. The use of our newly proposed IS for quantification of HGs

135 in samples from the natural environment should be of benefit vs. e.g. external

136 standards or ISs with a different polarity and structure (such as the platelet

137 activating factor (PAF) standard (1-O-hexadecyl-2-acetyl-sn-glycero-3-

138 phosphocholine; Lipp and Hinrichs (2009); Moore et al. (2013)), as it allows

139 correction for sample loss, e.g. during pre-analysis filtration of samples or during

140 sample injection.

141 To test its application, the IS was used during UHPLC-HRMS analysis of

142 a microbial mat from a beach on the North Sea Dutch barrier island of

143 Schiermonnikoog. It was found to contain $22.7 \mathrm{ng} / \mathrm{g}$ dry sediment of $\mathrm{C}_{6} \mathrm{HG}_{26}$ diol.

144 For comparison, the microbial mat was found to contain $3.74 \mu \mathrm{g} / \mathrm{g}$ dry sediment 
145 of the sulfoquinovosyldiacylglycerol (SQDG) with fatty acid constituents 16:0 and

$146 \quad$ 18:3 (Bale et al., unpublished data).

147 Our method therefore allows quantification of HGs in biomass and

148 sediments and thereby quantitative comparison with other lipids and inference

149 of the importance of past heterocystous $\mathrm{N}_{2}$ fixation in ancient environments.

\section{Acknowledgments}

We would like to thank 2 anonymous reviewers for helpful comments. We

152 also thank L.J. Stal, M. Grego and L. Villanueva for providing the cyanobacterial

153 biomass, and H. Fan and L. J. Stal for assistance with, and collection of, the

154 microbial mat sample. The work of N.B. is supported by the Netherlands

155 Organisation for Scientific Research (NWO) through grant 822.01.017 to S.S.

and J.S.S.D. were financially supported by the Netherlands Earth Systems

157 Science Centre (NESSC), a Gravitation grant (024.002.001) from the Dutch

158 Ministry of Education, Culture and Science. J.S.S.D also receives funding from

159 the Soehngen Institute for Anaerobic Microbiology (SIAM) though a gravitation

160 grant from the Dutch ministry for Education, Culture and Science. Associate Editor - M. Elvert

\section{References}

163 Bale, N.J., Hopmans, E.C., Schoon, P.L., de Kluijver, A., Downing, J.A., Middelburg, J.J., Sinninghe Damsté, J.S., Schouten, S., 2016. Impact of trophic state on the distribution of intact polar lipids in surface waters of lakes. Limnology and Oceanography 61, 1065-1077.

167 Bale, N.J., Hopmans, E.C., Zell, C., Sobrinho, R.L., Kim, J.-H., Sinninghe Damsté, J.S., Villareal, T.A., Schouten, S., 2015. Long chain glycolipids 
with pentose head groups as biomarkers for marine endosymbiotic heterocystous cyanobacteria. Organic Geochemistry 81, 1-7.

Bauersachs, T., Compaore, J., Severin, I., Hopmans, E.C., Schouten, S., Stal, L.J., Sinninghe Damsté, J.S., 2011. Diazotrophic microbial community of coastal microbial mats of the southern North Sea. Geobiology 9, 349-359.

Bauersachs, T., Hopmans, E.C., Compaore, J., Stal, L.J., Schouten, S., Sinninghe Damsté, J.S., 2009. Rapid analysis of long-chain glycolipids in heterocystous cyanobacteria using high-performance liquid chromatography coupled to electrospray ionization tandem mass spectrometry. Rapid Communications in Mass Spectrometry 23, 13871394.

Bauersachs, T., Mudimu, O., Schulz, R., Schwark, L., 2014. Distribution of long chain heterocyst glycolipids in $\mathrm{N}_{2}$ fixing cyanobacteria of the order Stigonematales. Phytochemistry 98, 145-150.

Fan, H., Bolhuis, H., Stal, L.J., 2015. Nitrification and Nitrifying Bacteria in a Coastal Microbial Mat. Frontiers in Microbiology 6, 1367.

Gambacorta, A., Soriente, A., Trincone, A., Sodano, G., 1995. Biosynthesis of the heterocyst glycolipids in the cyanobacterium Anabaena cylindrica. Phytochemistry $39,771-774$.

Gambacorta, A., Trincone, A., Soriente, A., Sodano, G., 1999. Chemistry of glycolipids from the heterocysts of nitrogen-fixing cyanobacteria. Current Topics in Phytochemistry 2, 145-150.

Huguet, C., Hopmans, E.C., Febo-Ayala, W., Thompson, D.H., Sinninghe Damsté, J.S., Schouten, S., 2006. An improved method to determine the 
absolute abundance of glycerol dibiphytanyl glycerol tetraether lipids. Organic Geochemistry 37, 1036-1041.

Lipp, J.S., Hinrichs, K.-U., 2009. Structural diversity and fate of intact polar lipids in marine sediments. Geochimica et Cosmochimica Acta 73, 68166833.

Moore, E.K., Hopmans, E.C., Rijpstra, W.I.C., Villanueva, L., Dedysh, S.N., Kulichevskaya, I.S., Wienk, H., Schoutsen, F., Sinninghe Damsté, J.S., 2013. Novel mono-, di-, and trimethylornithine membrane lipids in Northern Wetland Planctomycetes. Applied Environmental Microbiology $79,6874-6884$.

Moore, E.K., Hopmans, E.C., Rijpstra, W.I.C., Villanueva, L., Sinninghe Damsté, 204 J.S., 2016. Elucidation and identification of amino acid containing

Schouten, S., Villareal, T.A., Hopmans, E.C., Mets, A., Swanson, K.M., Sinninghe Damsté, J.S., 2013. Endosymbiotic heterocystous cyanobacteria synthesize different heterocyst glycolipids than free-living heterocystous

212 Wörmer, L., Cires, S., Velazquez, D., Quesada, A., Hinrichs, K.-U., 2012. Cyanobacterial heterocyst glycolipids in cultures and environmental samples: Diversity and biomarker potential. Limnology and Oceanography $57,1775-1788$. 
216 Wörmer, L., Lipp, J.S., Schröder, J.M., Hinrichs, K.-U., 2013. Application of two new LC-ESI-MS methods for improved detection of intact polar lipids (IPLs) in environmental samples. Organic Geochemistry 59, 10-21.

219 Fig. 1 (a) UHPLC AM MS partial base peak chromatogram of mixture (1:1 w:w) 220 containing the IS and the two $\mathrm{C}_{6} \mathrm{HG}_{26}$ diol isomers ( $\mathrm{HG}$ and $\mathrm{HG}^{\prime}$ ). Insert: 221 structures of the IS (n-dodecyl-B-D-glucopyranoside) and $\mathrm{C}_{6} \mathrm{HG}_{26}$ diol. (b) $\mathrm{MS}^{2}$ 222 spectrum of IS. For the $\mathrm{MS}^{2}$ spectrum of $\mathrm{C}_{6} \mathrm{HG}_{26}$ diol, see Bauersach et al. 223 (2009). (c) Example of standard curves of weight on column vs. peak area for the 224 IS and the $\mathrm{C}_{6} \mathrm{HG}_{26}$ diol. 
a)

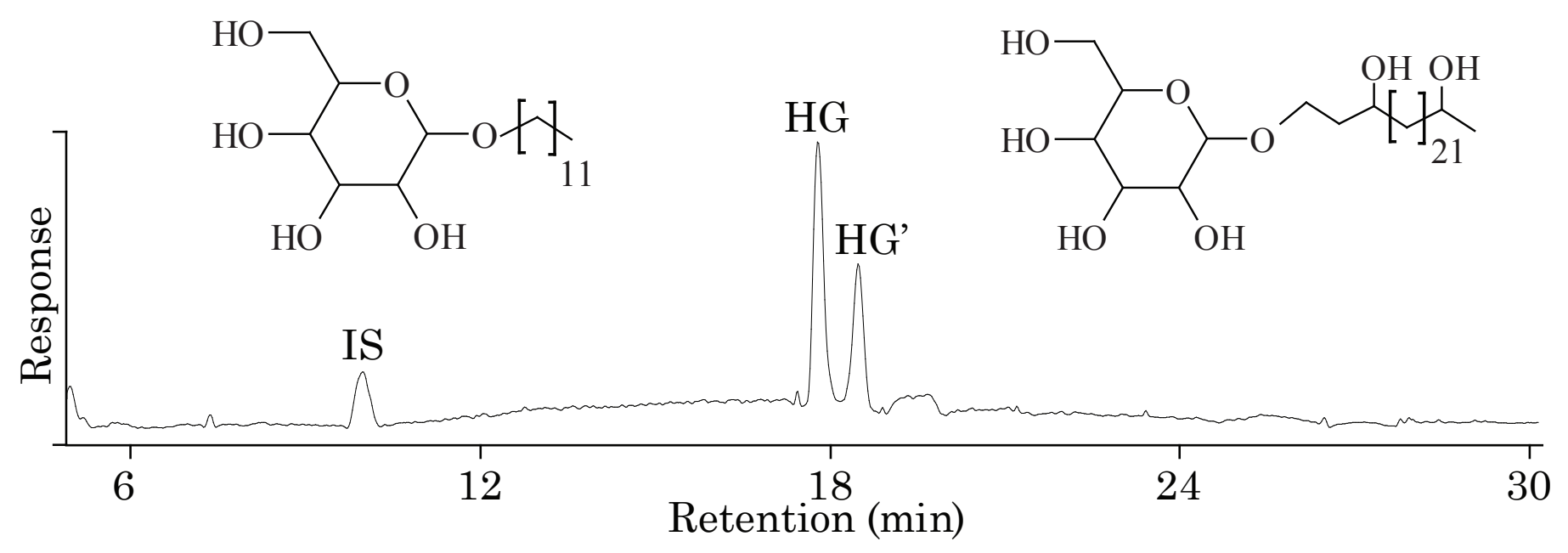

b)

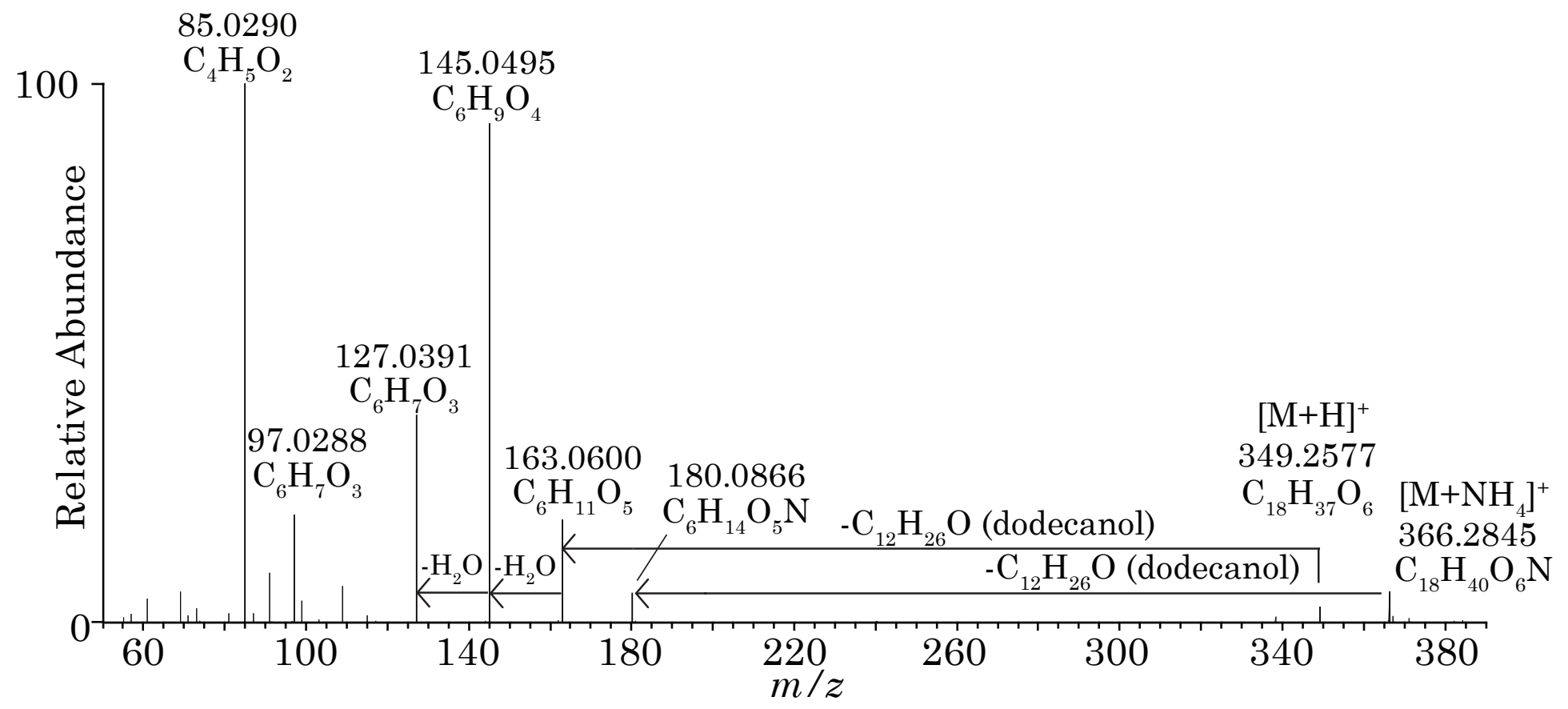

c)

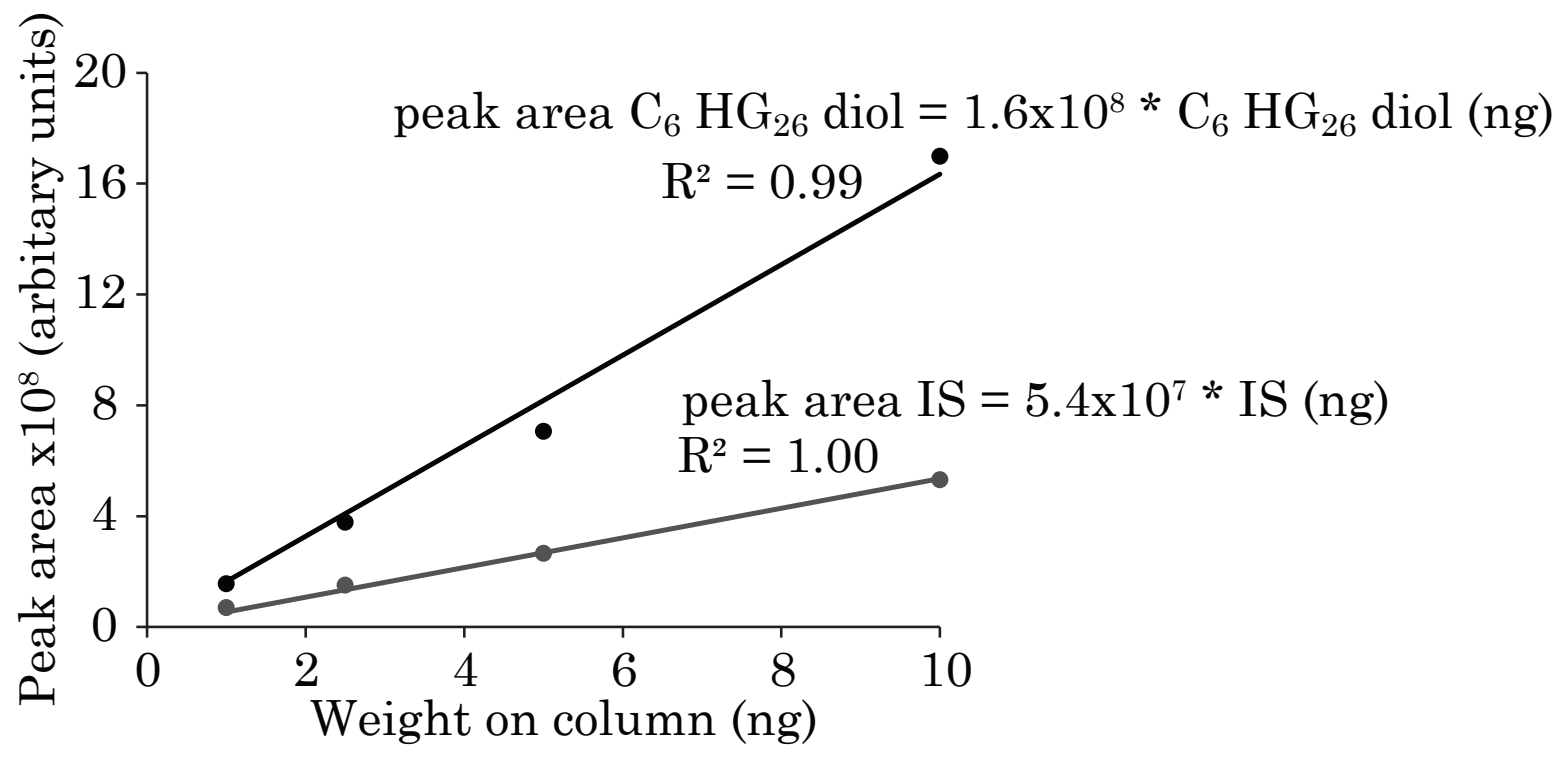

